\title{
Optofluidic waveguides written in hydrophobic silica aerogels with a femtosecond laser
}

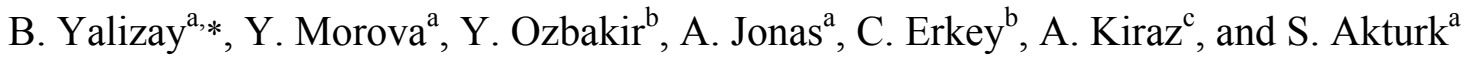 \\ ${ }^{a}$ Department of Physics, Istanbul Technical University, 34469 Maslak, Istanbul, Turkey; \\ ${ }^{b}$ Department of Chemistry, Koc University, Rumelifeneri Yolu, 34450 Sariyer, Istanbul, Turkey; \\ ${ }^{\mathrm{c}}$ Department of Physics, Koc University, Rumelifeneri Yolu, 34450 Sariyer, Istanbul, Turkey;
}

\begin{abstract}
We present a new method to form liquid-core optofluidic waveguides inside hydrophobic silica aerogels. Due to their unique material properties, aerogels are very attractive for a wide variety of applications; however, it is very challenging to process them with traditional methods such as milling, drilling, or cutting because of their fragile structure. Therefore, there is a need to develop alternative processes for formation of complex structures within the aerogels without damaging the material. In our study, we used focused femtosecond laser pulses for high-precision ablation of hydrophobic silica aerogels. During the ablation, we directed the laser beam with a galvo-mirror system and, subsequently, focused the beam through a scanning lens on the surface of bulk aerogel which was placed on a three-axis translation stage. We succeeded in obtaining high-quality linear microchannels inside aerogel monoliths by synchronizing the motion of the galvo-mirror scanner and the translation stage. Upon ablation, we created multimode liquid-core optical waveguides by filling the empty channels inside low-refractive index aerogel blocks with highrefractive index ethylene glycol. In order to demonstrate light guiding and measure optical attenuation of these waveguides, we coupled light into the waveguides with an optical fiber and measured the intensity of transmitted light as a function of the propagation distance inside the channel. The measured propagation losses of $9.9 \mathrm{~dB} / \mathrm{cm}$ demonstrate the potential of aerogel-based waveguides for efficient routing of light in optofluidic lightwave circuits.
\end{abstract}

Keywords: optofluidics, optical waveguides, microfluidics, aerogels, femtosecond laser ablation

\section{INTRODUCTION}

In recent years, optics and photonics research has been characterized by an increasing trend towards interdisciplinarity, synthesizing traditional optics with material science, chemistry, or biology. Optofluidics is one of the developing interdisciplinary areas of photonics that combines optics and microfluidics. Fluids have specific properties that cannot be found in solid materials, including straightforward adjustability of refractive indices, flexibility in shape and smooth interfaces. These properties can be used to design novel devices such as optofluidic microscope (OFM), dye lasers, optical trapping and manipulation systems, and chemical and biological sensors ${ }^{1-4}$.

One of the most important applications of optofluidic platforms is in light guiding. The overwhelming majority of conventional optical waveguides exploit total internal reflection (TIR). Waveguides based on TIR have a high refractive index $\left(n_{c}\right)$ core region surrounded by low index $\left(n_{s}\right)$ cladding materials (Fig. 1a). A light wave propagating in the waveguide core that is incident on the core-cladding interface at an angle $\theta$ above the critical angle, $\theta_{c}=\sin ^{-1} n_{s} / n_{c}$, remains confined in the core and propagates along the longitudinal direction (z). This principle is also known as index guiding. Index guiding can be implemented for various types of waveguides including solid core, liquid core, nanoporous cladding, liquid-liquid core (L2), and slot waveguides with different cross section geometries ${ }^{6}$ (Fig. $1 \mathrm{~b}$-f). In general, it is easy to provide the cladding material fulfilling the TIR condition for solid-core waveguides. However, the refractive index of water $(\sim 1.33)$ or other liquids which can be used to form the liquid core of optofluidic waveguides is typically lower than the refractive indices of commonly used solid materials required to confine the liquid within a microfluidic chip (polymer, glass, and silicon-based materials with $n_{s}=1.4-3.5$ ). Thus, it is rather challenging to produce TIR-based liquid core waveguides.

*yalizay@itu.edu.tr; phone +90 212285 3215; fax +90 2122856386

Integrated Optics: Devices, Materials, and Technologies XIX, edited by Jean-Emmanuel Broquin,

Gualtiero Nunzi Conti, Proc. of SPIE Vol. 9365, 936518 - (c) 2015 SPIE

CCC code: $0277-786 \mathrm{X} / 15 / \$ 18 \cdot$ doi: $10.1117 / 12.2077132$

Proc. of SPIE Vol. 9365 936518-1 
Cladding materials with internal coating of Teflon-like amorphous fluoropolymers (Teflon-AF) are one of the most common approaches for obtaining the TIR condition in optofluidic waveguides with aqueous liquid core ${ }^{7-11}$. Teflon-AF materials are optically transparent and have refractive index of 1.29-1.31, slightly lower than the refractive index of water (1.33). However, Teflon-AF has poor adhesion to commonly-used microfluidic chip materials and the low contrast of refractive index between Teflon-AF and water limits the efficiency of light confinement within the waveguide. Also, coating the cladding material with Teflon-AF is considerably costly even with a small thickness of the applied layers ${ }^{12}$. Hence, alternative solid cladding materials with a low index of refraction are currently sought for optofluidic applications utilizing liquid-core waveguides.
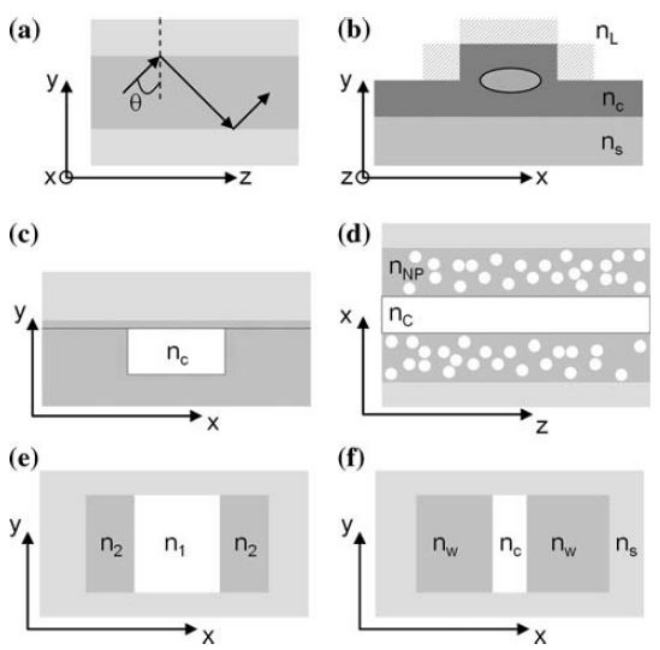

Figure 1: TIR based optical waveguides. (a) Principle of TIR. (b) Cross section of solid core waveguide. (c) Cross section of liquid core waveguide. (d) Side view of waveguide with nanoporous cladding material. (e) Liquid-liquid (L2) core waveguide cross section. (f) Slot waveguide cross section ${ }^{6}$. Figure adopted from Ref. [6].

Aerogels are nanoporous materials which have extremely low refractive index and thermal conductivity, high surface area to volume ratio, and high optical transparency in comparison to solid matter or normal foams. Additionally, aerogels have characteristic gel-like structural properties with nanoscale coherent skeletons and pores, hierarchical and fractal microstructure, and they are capable of forming macroscopic monoliths ${ }^{13}$. Thus, they represent an attractive choice for building optofluidic devices and systems. Aerogels were first produced by Kistler in $1931^{14}$ and since then, they have found a wide variety of applications exploiting their unique material properties. Table 1 summarizes the properties of a typical silica aeroge $1^{15}$ and Figure 2 shows examples of monolithic aerogel blocks.

Table 1. Properties of typical silica aerogels.

\begin{tabular}{|l|l|}
\hline \multicolumn{1}{|c|}{ Property } & \multicolumn{1}{c|}{ Value } \\
\hline Density & $0.03-0.35 \mathrm{~g} / \mathrm{cm}^{3}$ \\
\hline Internal surface area & $600-1000 \mathrm{~m}^{2} / \mathrm{g}$ \\
\hline Solid rate & $0.13 \%-15 \%$ \\
\hline Mean pore diameter & $\sim 20 \mathrm{~nm}$ \\
\hline Particle diameter & $2-5 \mathrm{~nm}$ \\
\hline Refractive index & $1.0-1.08$ \\
\hline Coefficient of thermal expansion & $2.0-4.0 \times 10^{-6}$ \\
\hline Dielectric constant & $\sim 1.1$ \\
\hline Sound velocity & $100 \mathrm{~m} / \mathrm{s}$ \\
\hline
\end{tabular}


Microstructural properties of aerogels depend strongly on the aerogel synthesis procedure which, therefore, needs to be carefully controlled. In general, there are three basic steps for synthesis of silica aerogels ${ }^{13}$. The first one is the solution sol transition. In this stage, nanoscale sol particles are generated through spontaneous or catalyzed hydrolysis and condensation reactions in the precursor solution. In the second stage, the sol - gel transition, the sol particles create gellike structure by assembling hierarchically into a wet gel. The last step is the gel - aerogel transition (drying) when the solution inside the wet gel is replaced by air without corrupting the solid structure of the gel. In the last step, various drying methods can be used including high temperature supercritical drying, low temperature supercritical drying, natural drying, and freezing drying ${ }^{16,17}$. Depending on the synthesis methods, aerogels can have hydrophilic or hydrophobic surfaces. According to the intended application, wetting properties of silica aerogels becomes important. For example, hydrophilic aerogels are not applicable for microfluidic applications with aqueous solutions because hydrophilic aerogel monoliths completely collapse when they come into contact with water. In addition, aerogels become opaque with increasing density of the solid skeleton (compare Figure 2a and 2b). Aerogel density can be adjusted by controlling the concentration of aerogel precursor in the original solution and by regulating synthesis conditions.
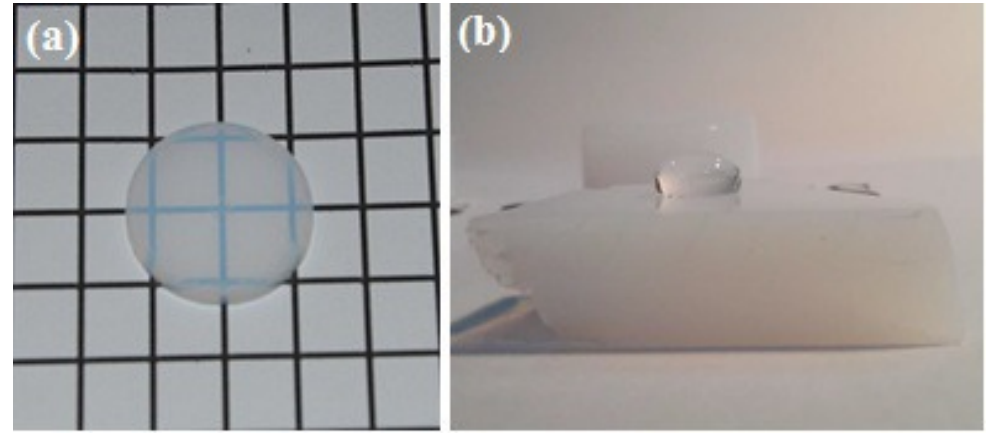

Figure 2: (a) Image of transparent silica aerogel with low density. (b) Image of high-density opaque hydrophobic silica aerogel.

Ultra-low index of refraction and high transparency make hydrophobic aerogels attractive materials for optofluidic waveguiding applications. In order to integrate these remarkable materials to the optofluidic systems, there is a need to process the aerogels. However, it is very challenging to process them with traditional machining methods such as milling, drilling, or cutting because of their fragile structure ${ }^{18}$. Inserting suitably shaped molds into the aerogel precursor solution during the gelation phase of aerogel synthesis is a successful method for creating linear or U-shaped microchannels inside aerogel monoliths ${ }^{19,20}$. However, this technique is not suitable for the formation of more complicated internal structures. Therefore, there is a need to develop alternative manufacturing processes for formation of complex structures within the aerogels without damaging the material. In this context, femtosecond laser micromachining is one of the most promising techniques for creating microstructures on the surface or in the bulk of delicate materials like aerogels.

Femtosecond lasers produce pulses with a duration well below $1 \mathrm{ps}$, i.e. in the time domain of tens to hundreds of femtoseconds $\left(1 \mathrm{fs}=10^{-15} \mathrm{~s}\right)$. Such short pulses are generally created by the technique of mode locking which induces a fixed-phase relationship between the modes of the laser resonant cavity. Thus, lasers outputting fs-pulses are also called mode-locked or phase-locked lasers ${ }^{21}$. As argued below, ultrafast laser pulses are particularly well suited for precise high-resolution processing of optically transparent materials.

In general, laser ablation of materials occurs in several steps following the interaction of focused light pulses with the material on different time scales ${ }^{22,23}$. Immediately upon the incidence of the pulse, electrons undergo nonlinear ionization and the material within the optical penetration depth is ablated through electron emission, sublimation, and plasma transition. This process occurs on the sub-picosecond time scale. Within several picoseconds, heat transfer between the excited electrons and ions of the material lattice takes place, resulting in lattice heating and melting. Heating of the lattice then causes a shock wave that starts propagating through the material within nanoseconds, accompanied by heat diffusion from the ablation zone. In applications with long laser pulses, thermally induced tension and propagating shock waves cause changes of material characteristics and structure manifested by the formation of micro-cracks, surface damage and surface contamination with ablation debris (Figure 3, left). However, when ultrafast laser pulses are employed, none of these unfavorable thermal effects are observed since the excitation pulse ends before the "hot" electrons have a chance to transfer their thermal energy to the lattice. Thus, heat diffusion outside of the beam focal area 
is minimized which leads to a more precise and clean micromachining. Moreover, because of its strongly non-linear character, interaction between ultrafast light and material is confined to the high-intensity region in the immediate vicinity of the beam focus which further contributes to the high resolution and precision of the laser ablation (Figure 3, right). An additional advantage of material processing with femtosecond lasers is then the ability to work with many materials ranging from optically transparent dielectric materials to metals. Recent studies of ultrafast-light micromachining of silica aerogels have shown that such aerogels can be precisely ablated with femtosecond lasers, thanks to their ultra-low thermal conductivity ${ }^{24}$.
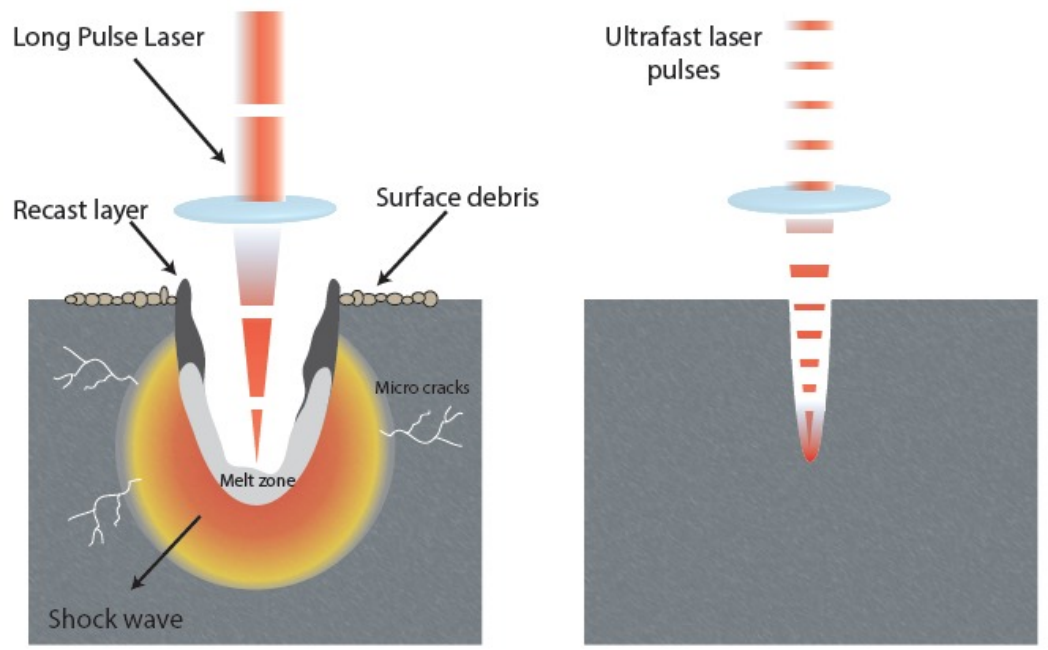

Figure 3: The effects of interaction between a solid material and a long laser pulse (left) or an ultrafast laser pulse (right) ${ }^{25}$.

Figure originated from Ref. [25]

In this study, we introduce TIR-based liquid-core optofluidic waveguides manufactured by direct femtosecond laser writing of void channels inside aerogel monoliths. Our waveguides use low-refractive index aerogels processed by laser ablation as the solid cladding and ethylene glycol filaments confined within the aerogels as the high-refractive index liquid cores. We present an experimental procedure for manufacturing optofluidic waveguides of millimeter length. Subsequently, we characterize the light-guiding properties of our waveguides and show that they can serve for efficient light routing in integrated optofluidic systems.

\section{FABRICATION OF AEROGEL-BASED OPTOFLUIDIC WAVEGUIDES}

\subsection{Aerogel synthesis}

We used a two-step sol-gel process to prepare aerogel samples ${ }^{19,26}$. In this process, silica precursor was Tetraethylorthosilicate (TEOS) (98\% purity; AlfaAesar), acid catalyst was hydrochloric acid ( $\mathrm{HCl})(37 \%$ purity; Riedelde Haen), and base catalyst was ammonia $\left(\mathrm{NH}_{3}\right)(2.0 \mathrm{M}$ in ethanol; Aldrich). In the beginning, we prepared the precursor solution by mixing TEOS, ethanol (99.9\% purity; Merck) and water with a mass ratio 1:1:0.34 and adding the acid catalyst for accelerating hydrolysis reactions. Subsequently, base catalyst was added to promote condensation and gelation. After gelation, we placed the resulting alcogels in an aging solution with $120 \mathrm{ml}$ TEOS: $30 \mathrm{ml}$ water: $150 \mathrm{ml}$ ethanol and kept the alcogels in this solution for several days. In the final step, we dried the alcogels at $40^{\circ} \mathrm{C}, 100$ bar with supercritical $\mathrm{CO}_{2}(\mathrm{scCO} 2)$. After the drying stage, we obtained monolithic aerogel samples (see Figure 2).

\subsection{Femtosecond laser ablation}

In order to generate a microchannel inside a hydrophobic silica aerogel monolith, we used the experimental setup shown in the top part of Figure 4. The laser (s-Pulse; Amplitude Systems) produced pulses with 550 fs duration and $1 \mathrm{kHz}$ repetition rate at $1030 \mathrm{~nm}$ wavelength. The average power of the ablation beam could be controlled using a combination of a half-wave plate and a polarizer. We ablated the sample with a maximum laser power of $200 \mathrm{~mW}$ at the specimen. During the ablation, we adjusted the beam angle with a dual-axis galvo system (ThorLabs, GVS012) that was controlled by a two-channel signal generator and focused the beam on the surface of the aerogel monolith through a scan lens 
(ThorLabs, LSM03-VIS). The diameter of the focused laser beam was about $10 \mu \mathrm{m}$. To create the channel, we moved the sample along the optical axis (z-direction) with a three-dimensional translation stage.

The microchannel with a desired cross-section was created in two steps. Initially, we sent sinusoidal signals with the same frequency and a phase difference of $90^{\circ}$ to both $\mathrm{x}$ - and y-axis of the galvo scanner, resulting in circular motion of the ablation beam focus. This circular pattern defined the channel outline. Subsequently, we sent sinusoidal signals of different frequencies to the $\mathrm{x}$ - and $\mathrm{y}$-axis of the beam scanner, obtaining a Lissajous pattern which scanned the inner parts of the channel cross-section. Figure 4, bottom, shows the overall two-step ablation pattern. Simultaneously with the $\mathrm{x}, \mathrm{y}$-motion of the galvo system, we translated the sample along the z-axis with a speed of $10 \mu \mathrm{m} / \mathrm{s}$. In this way, we formed cylindrical microchannels with $\sim 5 \mathrm{~mm}$ length and $\sim 500 \mu \mathrm{m}$ diameter inside the silica aerogel.

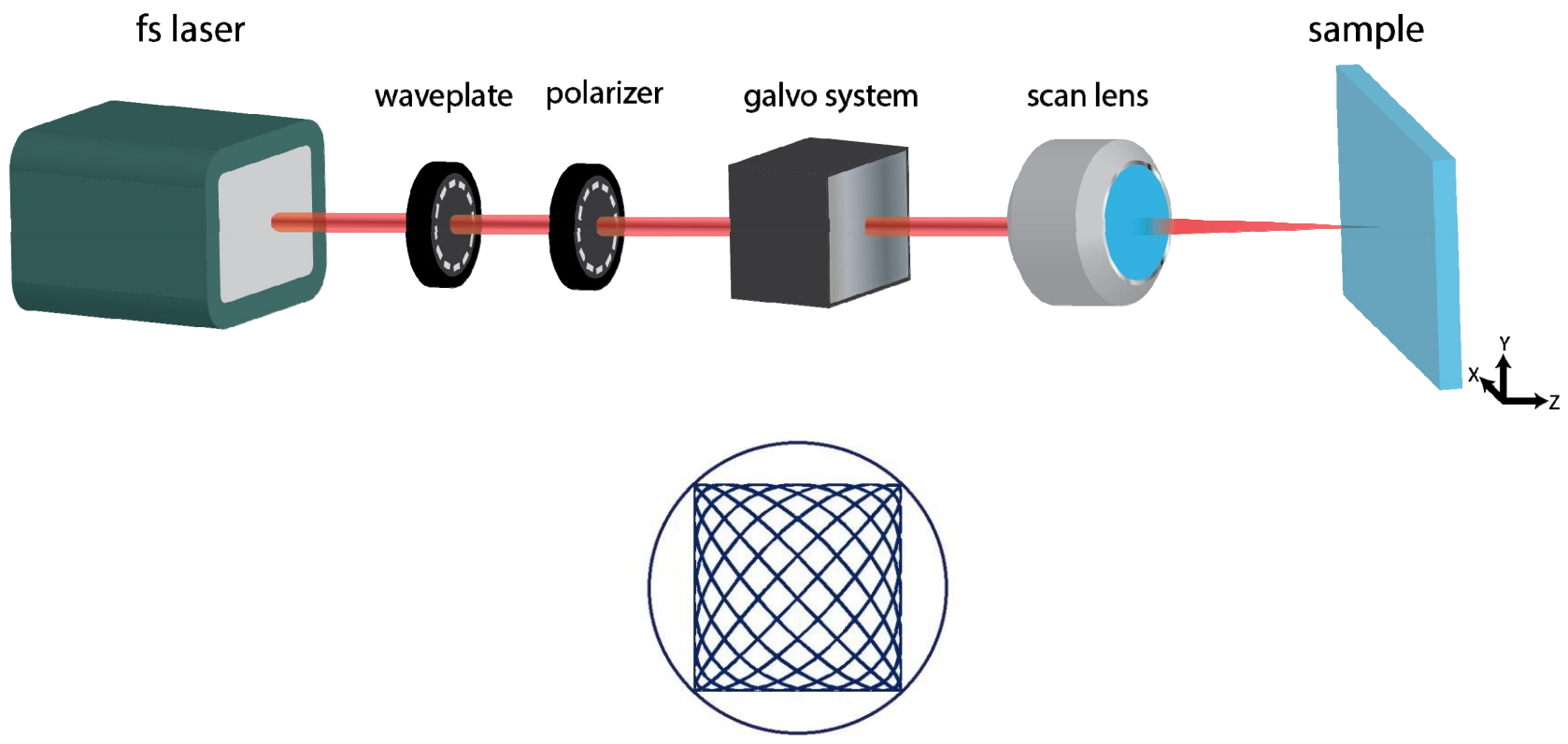

Figure 4. (top) Illustration of setup used in the aerogel ablation experiments (bottom) xy-scanning pattern of the ablation beam

\section{RESULTS AND DISCUSSION}

\subsection{Characterization of ablated aerogel channels}

In previous works, it has been shown that structural changes in aerogels can be achieved by femtosecond laser pulses ${ }^{24}$. In the present study, we provide direct experimental evidence of clean and precise micromachining of silica aerogels. Using femtosecond laser writing, we were able to form high-quality linear microchannels inside hydrophobic silica aerogels.

Figure 5 presents scanning electron microscope (SEM) images of aerogel microchannels prepared by the above described procedure. In Fig. 5a, input face of the channel is depicted, whereas Figs. 5b and 5c show the axial crosssections of the channels. One of the most important factors that affect the maximal length of the channel that can be manufactured is the accumulation of ablated silica particles within the channel during the sample processing. When the laser ablation is carried out close to the monolith surface, the ablated aerogel particles can escape into air. However, when we ablate deeper in the aerogel, accumulation of the silica particles inside the channel can be observed (see Fig. $5 b$ ). These particles cause scattering of the ablation laser beam and limit the maximal depth of the channel that can be produced. In order to prevent this phenomenon, we exposed the sample to directed flow of high pressure $(\sim 4 \mathrm{~atm})$ nitrogen gas during the ablation. Figure 5c shows SEM image of the axial cross-section of a channel which was formed with nitrogen gas treatment. Comparison of Figs. $5 \mathrm{~b}$ and $5 \mathrm{c}$ shows a clear improvement in the uniformity of the channel 
axial profile for nitrogen-treated samples, with all silica dust particles successfully removed by the nitrogen flow. Using this method, we succeeded in machining channels with the total length up to $5 \mathrm{~mm}$.
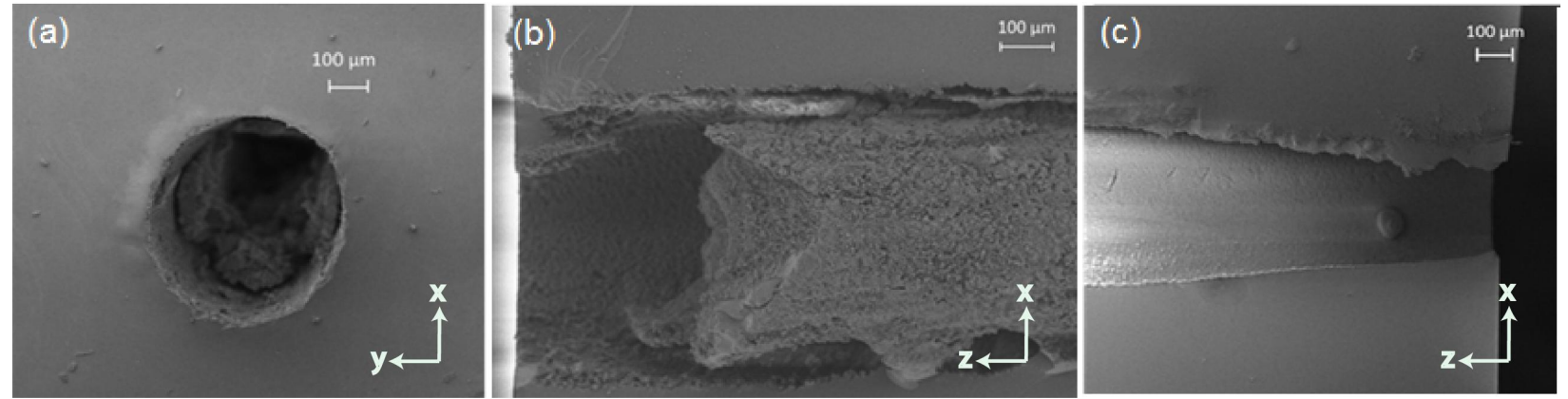

Figure 5. SEM images of ablated aerogels. (a) Input face of a microchannel ablated inside the aerogel. The depth of the channel is about $300 \mu \mathrm{m}$ and the diameter is about $500 \mu \mathrm{m}$. (b) Axial cross-section view of a channel machined inside the aerogel. During machining, ablated silica particles accumulated inside the channel. (c) Axial cross-section view of a channel ablated with simultaneous purging of the sample with high-pressure nitrogen gas. All the dust created inside the channel during machining has been removed. For recording the cross-sectional images shown in (b) and (c), the samples were cut along the axis of the channel.

\subsection{Characterization of light-guiding performance of liquid-core optofluidic waveguides}

After successful ablation of void microchannels inside the aerogel monoliths, we generated liquid-core optofluidic waveguides by filling the channels with ethylene glycol. Ethylene glycol has refractive index of 1.43 and its polar character ensures stable confinement inside the aerogel channels with hydrophobic walls. The advantage of ethylene glycol over water is its lower volatility and, thus, slower evaporation rate, which extends the operation time of the waveguides. To visualize liquid confinement within the channel, we mixed ethylene glycol with fluorescein, an organic dye with peak excitation at $494 \mathrm{~nm}$ and peak emission at $521 \mathrm{~nm}$. When illuminated with a blue $(405 \mathrm{~nm})$ laser light, sharply localized green fluorescence from the channel was clearly visible (see Fig. 6). This illustrates an excellent leakfree confinement of ethylene glycol within the channel which is a prerequisite for stable operation of the waveguide.

Figure 7 illustrates experimental arrangement used for the characterization of light-guiding properties of our optofluidic waveguides and measurement of their propagation losses. To this end, we coupled the light from an auxiliary diode laser (wavelength $632.8 \mathrm{~nm}$ ) into the waveguide core through a tapered optical fiber with an effective numerical aperture (NA) of 0.57 . To quantify the amount of light transmitted through the waveguide, we imaged the exit face of the channel on a CCD camera (ThorLabs DCU223M) using a microscope objective in combination with a projection lens.

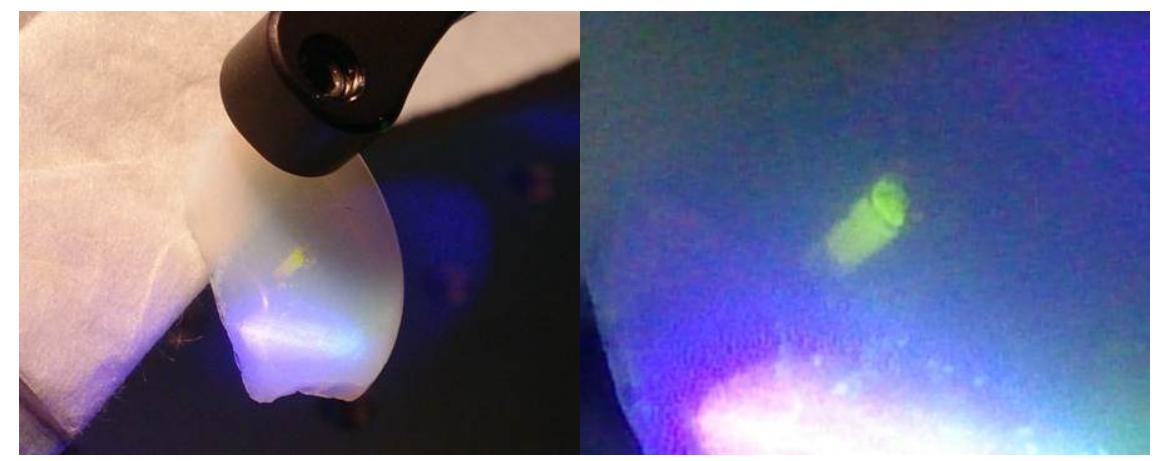

Figure 6: Green fluorescence from the aerogel channel filled with fluorescein solution in ethylene glycol upon illumination with blue light. 


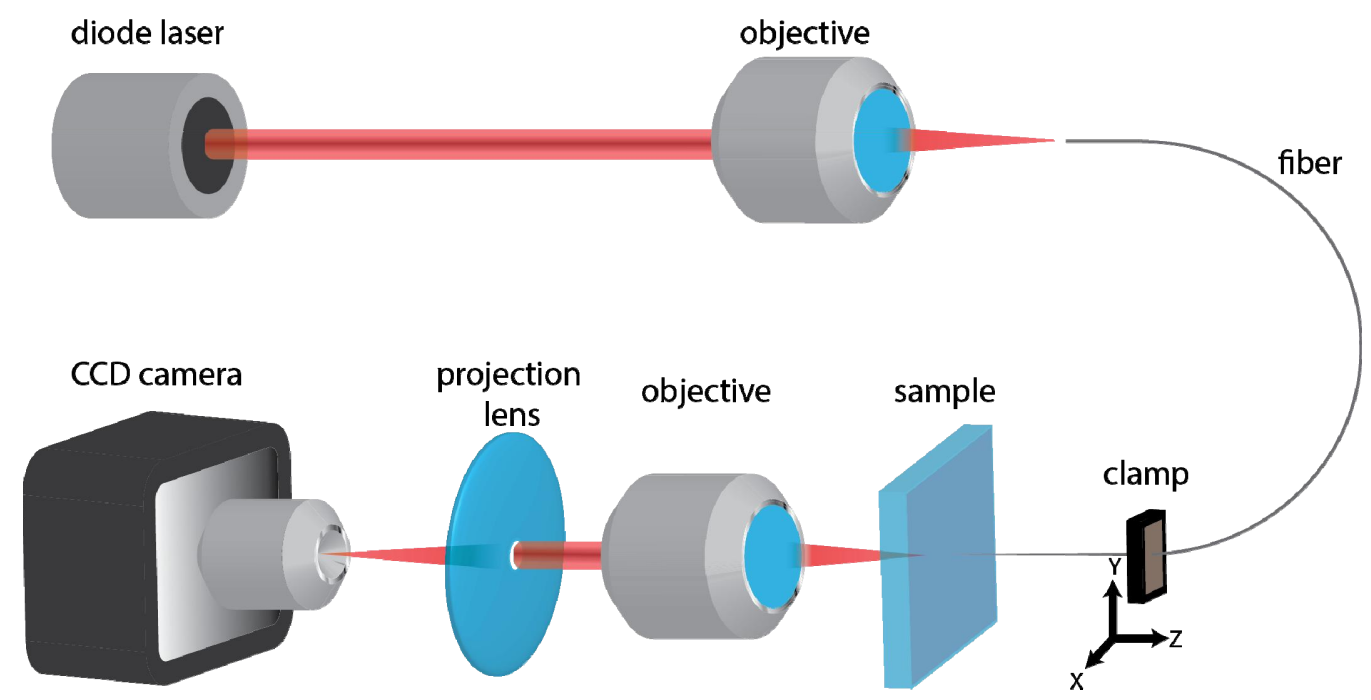

Figure 7: Schematics of experimental setup used in the characterization of light-guiding performance of liquid core optofluidic waveguides.

We measured the diameter of the channel to be about $600 \mu \mathrm{m}$ and the NA of the input coupling fiber to be approximately 0.57. Hence, we calculated the beam diverging from the end of the fiber reaches the walls of the channel after propagating $1.1 \mathrm{~mm}$ along the channel axis. After this distance, the light fulfills the TIR condition and propagates inside the channel.

In order to measure the propagation losses of the waveguide, we moved the coupling fiber along the channel axis and, simultaneously, monitored the total intensity of light exiting the channel. This was achieved by summing the grayscale values of all pixels in the image of the waveguide exit face. An illustration image of the guided laser light can be seen in Figure 8. Recorded light distribution covering the entire channel cross-section indicates that the coupled light was confined and guided inside the channel. Because of the multimode behavior of our large-core waveguides, the output light distribution features a characteristic speckle pattern.

We recorded the images of the output intensity distribution for $100 \mu \mathrm{m}$ increments of the input fiber position along the channel axis. Subsequently, we plotted the total transmitted intensity as a function of the propagation distance along the waveguide. The results of this series of measurements are shown in Figure 9. As illustrated in this figure, the measured output intensity of the waveguide displays regular oscillations superimposed on an exponentially decreasing background. These oscillations can be attributed to beating of multiple guided modes that propagate in the channel and whose relative phases change as the input fiber is translated along the waveguide axis.

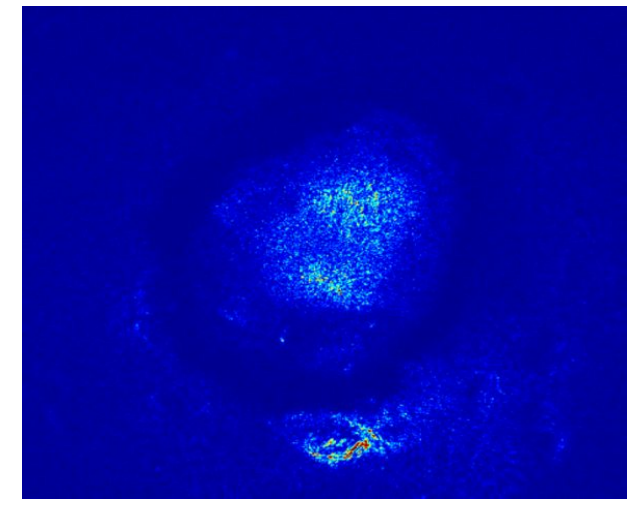

Figure 8: Pseudo-color CCD image of guided light in the liquid-core waveguide inside the aerogel. 


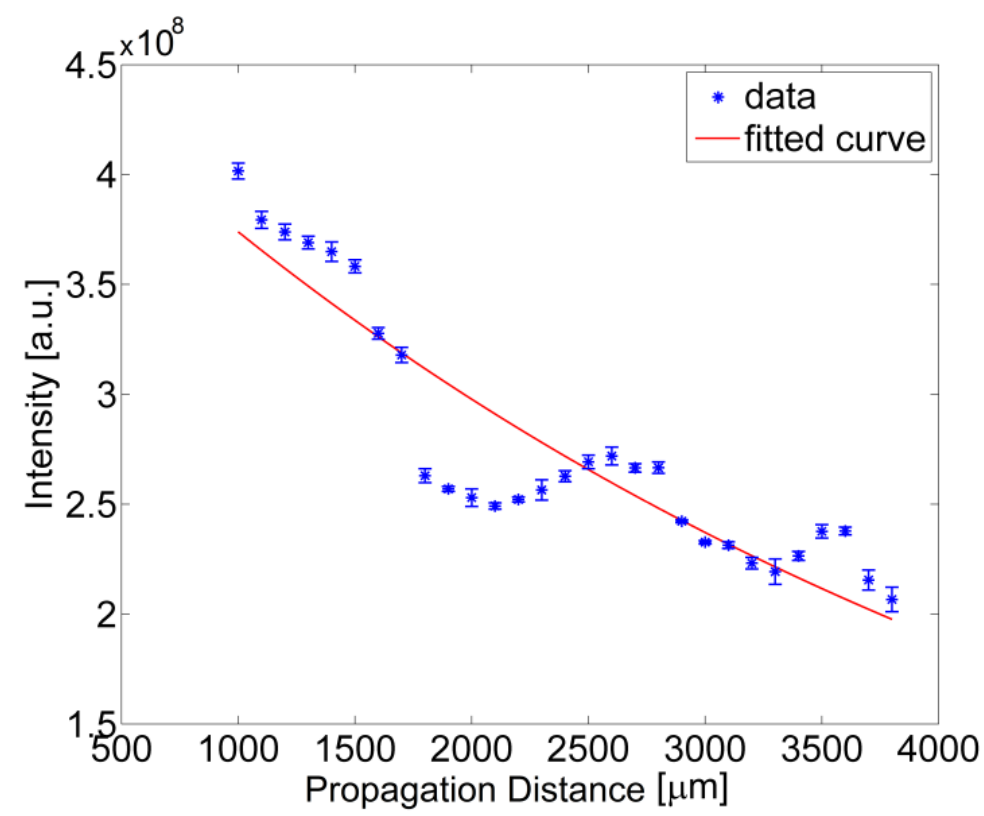

Figure 9: Intensity of light transmitted through the waveguide versus the propagation distance along the waveguide axis.

To quantify the propagation losses of the waveguide, we fitted the experimental values of the transmitted light intensity with an exponential curve of the form $I=I_{0} \exp (-\alpha z)$ where $\mathrm{z}$ is the propagation distance along the waveguide axis. For the particular data set shown in Fig. 9, the calculated value of $\alpha=2.278 \mathrm{~cm}^{-1}$. Hence, the propagation loss of the waveguide in the units of $\mathrm{dB} / \mathrm{cm}$ can be calculated from

$$
\eta=-\frac{10 \alpha}{\ln 10}
$$

According to Eq. (1), $\eta=-9.9 \mathrm{~dB} / \mathrm{cm}$. This value is comparable to the propagation losses measured previously for alternative liquid-core optofluidic waveguide platforms ${ }^{8,27}$.

\section{CONCLUSION}

We have demonstrated the fabrication of liquid-core optofluidic waveguides based on void channels created inside hydrophobic silica aerogels by femtosecond laser micromachining and subsequently filled with ethylene glycol. We have investigated light-guiding properties of thus manufactured waveguides and measured their optical propagation losses. The experimentally obtained light-guiding characteristics show the potential of aerogel-based waveguides for efficient routing of light in optofluidic lightwave circuits. Even though we have studied straight optofluidic waveguides in the present work, femtosecond ablation enables fabrication of arbitrary three-dimensional paths within the transparent silica aerogels, thus expanding significantly the application range of aerogel-based optofluidics. Experiments with manufacturing curved liquid-core optofluidic waveguides and additional elements of photonic circuits (e.g. Y-junctions) are currently underway.

\section{REFERENCES}

[1] Grujic, K., Hellesø, O. G., Hole, J. P.., Wilkinson, J. S., "Sorting of polystyrene microspheres using a Ybranched optical waveguide," Opt. Express 13(1), 1-7 (2005).

[2] Mandal, S., "Optofluidic Transport - Sudeep Mandal's Website,"

$<$ http://www.sudeepmandal.com/research/optofluidic-transport/> (26 November 2014 ). 
[3] Schmidt, H.., Hawkins, A. R., "The photonic integration of non-solid media using optofluidics," Nat. Photonics 5(10), 598-604 (2011).

[4] Fan, X.., White, I. M., "Optofluidic microsystems for chemical and biological analysis," Nat. Photonics 5(10), 591-597 (2011).

[5] Measor, P., Kühn, S., Schmidt, H., Phillips, B., Lunt, E.., Hawkins, A., “New chip miniaturizes particle manipulation, detection," SPIE Newsroom (2011).

[6] Schmidt, H.., Hawkins, A. R., "Optofluidic waveguides: I. Concepts and implementations," Microfluid. Nanofluidics 4(1-2), 3-16 (2008).

[7] Cho, S. H., Godin, J.., Lo, Y.-H., "Optofluidic Waveguides in Teflon AF-Coated PDMS Microfluidic Channels," IEEE Photonics Technol. Lett. 21(15), 1057-1059 (2009).

[8] Manor, R., Datta, A., Ahmad, I., Holtz, M., Gangopadhyay, S.., Dallas, T., "Microfabrication and characterization of liquid core waveguide glass channels coated with Teflon AF," IEEE Sens. J. 3(6), 687-692 (2003).

[9] Altkorn, R., Koev, I.., Gottlieb, A., “Waveguide Capillary Cell for Low-Refractive-Index Liquids,” Appl. Spectrosc. 51(10), 1554-1558 (1997).

[10] Datta, A., Eom, I.-Y., Dhar, A., Kuban, P., Manor, R., Ahmad, I., Gangopadhyay, S., Dallas, T., Holtz, M., et al., "Microfabrication and characterization of teflon AF-coated liquid core waveguide channels in silicon," IEEE Sens. J. 3(6), 788-795 (2003).

[11] Wu, C.-W.., Gong, G.-C., "Fabrication of PDMS-Based Nitrite Sensors Using Teflon AF Coating Microchannels," IEEE Sens. J. 8(5), 465-469 (2008).

[12] Nath, G., "Tube having amorphous fluorinated copolymer," US6314227 B1 (2001).

[13] Du, A., Zhou, B., Zhang, Z.., Shen, J., "A Special Material or a New State of Matter: A Review and Reconsideration of the Aerogel," Materials 6(3), 941-968 (2013).

[14] Kistler, S. S., "Coherent Expanded Aerogels and Jellies," Nature 127, 741 (1931).

[15] Soleimani Dorcheh, A.., Abbasi, M. H., "Silica aerogel; synthesis, properties and characterization," J. Mater. Process. Technol. 199(1-3), 10-26 (2008).

[16] Aegerter, M. A., Leventis, N.., Koebel, M. M., Aerogels Handbook, Springer (2011).

[17] Menaa, B., Menaa, F., Aiolfi-Guimaraes, C.., Sharts, O., "Silica-based nanoporous sol-gel glasses: from bioencapsulation to protein folding studies," Int. J. Nanotechnol. 7(1), 1-45 (2010).

[18] Parmenter, K. E.., Milstein, F., “Mechanical properties of silica aerogels,” J. Non-Cryst. Solids 223(3), 179-189 (1998).

[19] Eris, G., Sanli, D., Ulker, Z., Bozbag, S. E., Jonás, A., Kiraz, A.., Erkey, C., "Three-dimensional optofluidic waveguides in hydrophobic silica aerogels via supercritical fluid processing," J. Supercrit. Fluids 73, 28-33 (2013).

[20] Xiao, L.., Birks, T. A., "Optofluidic microchannels in aerogel,” Opt. Lett. 36(16), 3275-3277 (2011).

[21] Haus, H. A., "Mode-locking of lasers," IEEE J. Sel. Top. Quantum Electron. 6(6), 1173-1185 (2000).

[22] Chichkov, B. N., Momma, C., Nolte, S., Alvensleben, F. von., Tünnermann, A., "Femtosecond, picosecond and nanosecond laser ablation of solids," Appl. Phys. A 63(2), 109-115 (1996).

[23] Gattass, R. R.., Mazur, E., "Femtosecond laser micromachining in transparent materials," Nat. Photonics 2(4), 219-225 (2008).

[24] Sun, J., Longtin, J. P.., Norris, P. M., “Ultrafast laser micromachining of silica aerogels," J. Non-Cryst. Solids 281(1-3), 39-47 (2001).

[25] Clark-MXR, Inc., "Machining with Long Pulse Lasers," Clark-MXR Inc, 1999, $<$ http://www.cmxr.com/Education/Long.html >.

[26] Gutzov, S., Danchova, N., Karakashev, S. I., Khristov, M., Ivanova, J.., Ulbikas, J., "Preparation and thermal properties of chemically prepared nanoporous silica aerogels," J. Sol-Gel Sci. Technol. 70(3), 511-516 (2014).

[27] Jonáš, A., Yalizay, B., Akturk, S.., Kiraz, A., "Free-standing optofluidic waveguides formed on patterned superhydrophobic surfaces," Appl. Phys. Lett. 104(9), 091123 (2014). 